\title{
Studies on Z-Window Based FIR Filters
}

\author{
Rahul Pachauri, ${ }^{1}$ Rajiv Saxena, ${ }^{1}$ and Sanjeev N. Sharma ${ }^{2}$ \\ ${ }^{1}$ Department of Electronics \& Communication Engineering, Jaypee University of Engineering \& Technology, Guna 473226, India \\ ${ }^{2}$ Department of Electronics \& Communication Engineering, Samrat Ashok Technological Institute, Vidisha 464001, India
}

Correspondence should be addressed to Rahul Pachauri; pachauri.123@gmail.com

Received 7 June 2013; Accepted 29 July 2013

Academic Editors: I. Guler and C. S. Lin

Copyright (C) 2013 Rahul Pachauri et al. This is an open access article distributed under the Creative Commons Attribution License, which permits unrestricted use, distribution, and reproduction in any medium, provided the original work is properly cited.

As per classification of the window functions, the Z-windows are grouped in the category of steerable side-lobe dip (SSLD) windows. In this work, the application of these windows for the design of FIR filters with improved filter parameters has been explored. The numbers of dips with their respective positions in the side-lobe region have been compositely used to tailor the window shape. Filter design relationships have been established and included in this paper. Simultaneously, an application of these Z-window based FIR filters in designing two-channel quadrature mirror filter (QMF) bank has been presented. Better values of reconstruction and aliasing errors have been achieved in contrast to the Kaiser window based QMF bank.

\section{Introduction}

The finite impulse response (FIR) filters are one of the prominent building blocks used in various applications of digital signal processing. These filters have got more popularity than infinite impulse response (IIR) filters because of their inherent stability and linear phase characteristics [1]. The straightforward approach to design the FIR filters is truncating the ideal impulse response using windows. Careful selection of window function reduces the ripples introduced by truncation in the frequency response of these filters [2]. In the literature, several categories of windows have been proposed $[3,4]$. One of the important categories of windows is steerable side lobe dips (SSLD) windows in which deep dips can be steered in the side lobes. Tseng windows and Zhong windows (ZWs) are the examples of SSLD windows $[5,6]$. The ZW proposed by Zhong et al. [6] has simple expressions in either of the domains, good overall spectral characteristics, SSLD property and easy and flexible design technique as compared to Tseng window. These features of ZW motivated us to study this window from the FIR filter design perspective.

Prior to this work, the design of FIR filters using ZW has also been reported by Sharma et al. [7] in which filter order $(N)$ and transition bandwidth (TBW) have been minimized at the cost of higher passband ripple (PBR) and stopband attenuation (SBA). The filters designed by Sharma et al. [7] are having SBA in the range of $18-42 \mathrm{~dB}$ with single dip steered at an appropriate location in the side lobes of ZW. Followed by this work, the ZW filter (ZWF) has been designed using the traditional window based approach by Pachauri et al. [8] in which the PBR and SBA has been minimized at the cost of higher filter order. The expression of the filter order was modified in [8] and was made directly proportional to the number of SSLDs introduced in the ZW. It has also been demonstrated in [8] that the half main lobe width (HMLW) of ZW varies with dip displacement up to a certain position and after that it becomes constant. If the dips are introduced below this threshold position then the filter order reduces and PBR and SBA increase [7], whereas the filter order increases and $\mathrm{PBR}$ and SBA decrease when the dips are inserted on the other side of the threshold position [8]. With this background on spectral response of $\mathrm{ZW}$, the studies available in $[7,8]$, low pass filters have been designed using $\mathrm{Z}$-window in this work. Performance of these filters has been studied by varying number of dips in ZW along with respective variation in their position. This study helped us in establishing an application of this filter in multirate signal processing.

The remaining part of this paper has been organized as follows. In the next section ZW has been introduced and its properties from the filter design perspective have been discussed. In Section 3, an algorithm to establish the empirical design relationships for ZWF has been developed. Simulation results and the performance analysis of the proposed filters are included in Section 4. Design of quadrature mirror filter 
(QMF) bank using this filter as a prototype has been carried out in Section 5. A comparative study with Kaiser window (KW) based QMF bank has also been performed. Finally, the paper is concluded in Section 6.

\section{Characterization of $\mathrm{Z}$-Window for Filtering Application}

The basic concept in ZW proposed by Zhong et al. [6] is that the spectral window can be considered as a special LPF which only passes the DC component. The technique used for the designing of ZW is based on the frequency sampling method used for FIR filter design.

The ZW of length $N$ with $L$ SSLDs has been defined as [6]

$w(n)=\frac{1}{0.5+\sum_{k=1}^{L} x_{k}}\left[0.5+\sum_{k=1}^{L}(-1)^{k} x_{k} \cos \left(\frac{(2 n+1) \pi k}{N}\right)\right]$ for $n=0,1, \ldots, N-1$,

where,

$$
\begin{aligned}
& x_{k}= \cos \frac{\pi k}{N}\left[\prod_{l=1}^{L}\left(1-\cot ^{2} \frac{\omega_{l}}{2} \tan ^{2} \frac{\pi k}{N}\right)\right] \\
& \times\left(2\left[\prod_{i=1}^{k-1}\left(\cot ^{2} \frac{\pi i}{N} \tan ^{2} \frac{\pi k}{N}-1\right)\right]\right. \\
&\left.\cdot\left[\prod_{i=k+1}^{L}\left(1-\cot ^{2} \frac{\pi i}{N} \tan ^{2} \frac{\pi k}{N}\right)\right]\right)^{-1} \\
& \text { for } k=1,2, \ldots, L .
\end{aligned}
$$

In this window, instead of traditional approach to minimize the maximum side-lobe level (MSLL) with steepest descent or linear programming $[9,10]$, dips have been introduced at frequencies $\omega_{l}, l=1, \ldots, L$, masking the magnitude spectrum $\left|W\left(e^{j \omega}\right)\right|$ of the window at these frequencies.

A window of length $N$ may have $L$ dips which must satisfy the following conditions:

$$
\begin{gathered}
\pi>\omega_{l}>\frac{2 \pi L}{N}, \\
x_{1}>x_{2}>\cdots>x_{L}>0, \\
x_{k}<\frac{(L !)^{2}}{(L+k) ! \cdot(L+k) !}<1 .
\end{gathered}
$$

Condition (3) tells us that apart from finite set of frequencies: $\left(2 \pi(L+1) / N, \ldots, 2 \pi M / N\right.$, for $M=\left\{\begin{array}{cc}(N-1) / 2, & N \text { odd } \\ N / 2, & N \text { even }\end{array}\right)$, where zeros of $\left|W\left(e^{j \omega}\right)\right|$ already exist, $\omega_{l}$ can be assigned anywhere in the side lobes region, that is, $(2 \pi L / N, \pi)$. The meaningfulness of the solution is guaranteed by (4) and (5) as they give the upper bound of the solutions.

It has already been shown in [6] that by steering number of dips and their respective positions in the side lobes of ZW,

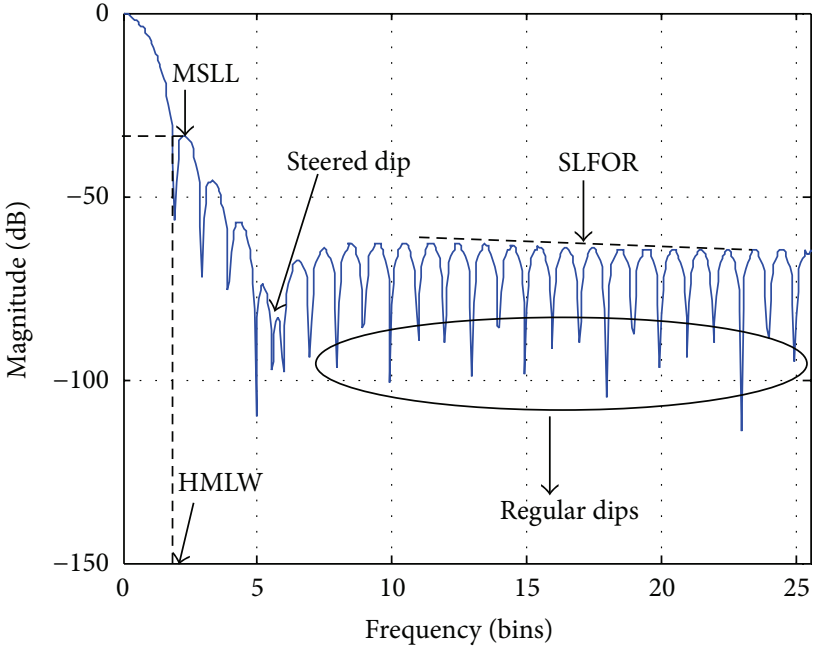

FIGURE 1: Plot for illustrating the definition of the various parameters of Z-window with $N=51, L=1$, and dip at 5.6.

the characteristics of the window, that is, HMLW, MSLL, and side lobe fall of rate (SLFOR), can be varied. To illustrate the above parameters, a ZW with one steerable dip is shown in Figure 1 . The normalized frequency in bins, which has been used to label the $x$-axis of the magnitude spectrum of ZW, has been defined as [11]

$$
\text { frequency in bins } f_{k}=\frac{k F_{s}}{N} \text { for } k=0,1, \ldots, N-1 \text {, }
$$

where $N$ is the window length and $F_{s}$ is the sampling frequency.

Other important properties of ZW given in $[6,8]$ and observed in this work are as follows.

(i) The dips can be steered in the side lobes anywhere between bins 1 and $N / 2$ [6].

(ii) The HMLW, MSLL, and SLFOR can be varied by varying the number of dips and their position in the side lobes. This follows from the simulation plots shown in Figures 2 and 3.

(iii) The HMLW increases linearly with the increase in position of dips initially and after that it becomes constant [8].

(iv) The MSLL decreases with position of dips, initially linearly, and after that it becomes almost constant. This behavior of MSLL is shown in Figure 4.

(v) It follows from Figures 1 and 2 that if the dips are inserted in the vicinity of $N / 2$ the HMLW and SLFOR are higher, while MSLL is higher than the minimum value which follows from Figure 4 . This property has been used in this work to obtain ZWF with maximum value of far end stopband attenuation (FESBA).

The above listed properties of Z-window function are directly associated with the design of FIR filters. Thus, 


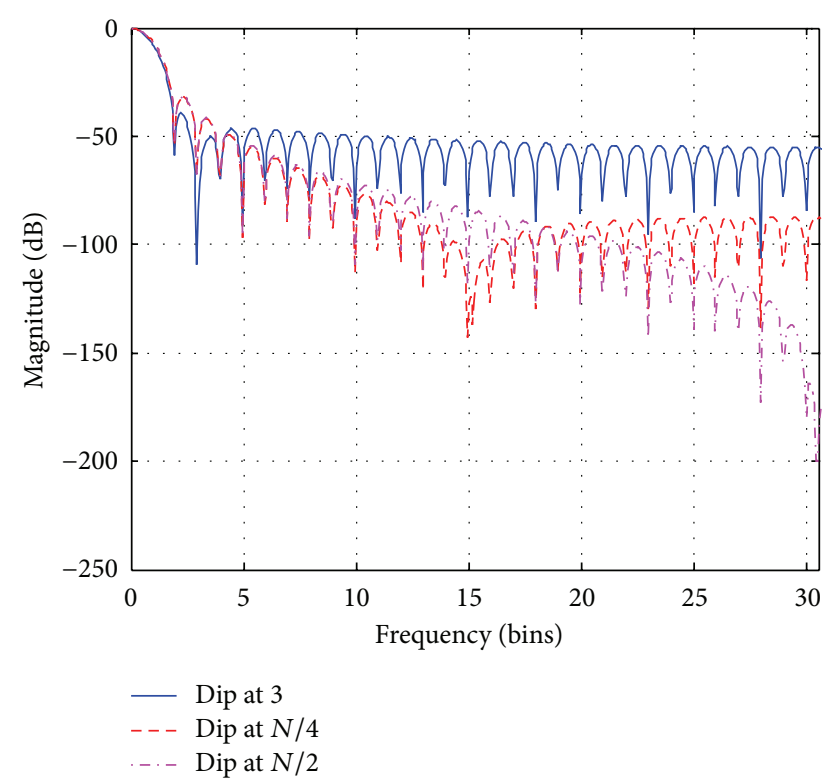

FIGURE 2: Variation of MSLL and SLFOR of ZW by insertion of one dip for a window of length $N=61$.

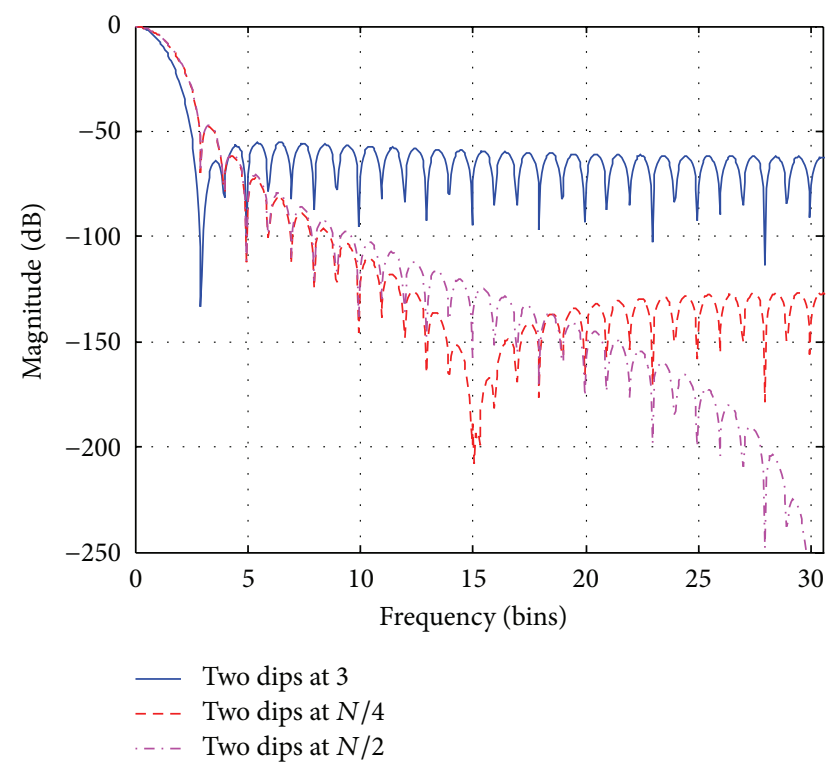

FIGURE 3: Variation of MSLL and SLFOR of ZW by insertion of two dips in a window of length $N=61$.

these properties of ZW have been exploited in this work to design FIR filters. In the proposed work, new empirical design relationships have been derived which provide more flexibility to vary the design parameters of ZWF, keeping desired specifications intact.

\section{Filter Design Relationships}

To design an FIR filter using window functions the following relationships are required [11].

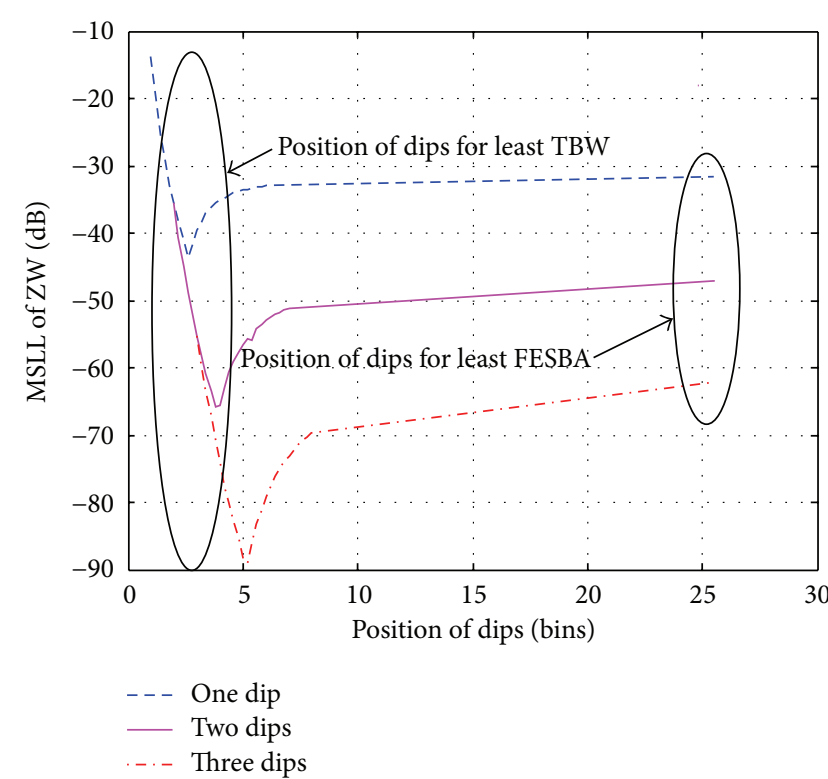

FIgURE 4: Variation of MSLL with position of dips for a window of length $N=51$.

(a) Relationship between $\mathrm{SBA}\left(A_{s}\right)$ and window shape parameter-this expression provides the value of window shape parameter for the desired SBA. In ZW, SSLD property, that is, number of dips $L$ and their position $D_{p}$, has been used to tailor the window shape.

(b) Expression defining the relationship between SBA $\left(A_{s}\right)$ and normalized window width parameter $(D)$ [12-14] - this value of $D$ is then used to calculate the filter order $N$ to provide the desired filter transition bandwidth (TBW; $\Delta f$ ), at a specified sampling frequency $\left(F_{s}\right)$. The value of $N$ is given by

$$
N \geq \frac{D}{\Delta f / F_{s}}+1
$$

To establish these relationships for the design of FIR low pass filters with $\mathrm{ZW}$, the parameters, namely, $L, D_{p}$, and $N$, were varied. The resulting variation in $A_{s}$ and $D$ is recorded in Table 1 and corresponding plots are shown in Figures 5 and 6. From these plots the empirical relations between $D, L, D_{p}$, and $A_{s}$ have been obtained. The procedure for establishing these relationships has been described with the aid of a flow chart shown in Figure 7. Conventionally, in window based FIR filter designs, filter order $N$ is initialized to a very large value and for this fixed value of $N$ design relationships are enumerated [12-14]. However, in this study, instead of using fixed $N$ it has been taken as a variable. Therefore, in this work variations in the values of $D$ and SBA have been obtained by varying the number of dips and their positions as well as the filter order $N$. The filter order and the number of dips have been used to change $D$ and $A_{s}$ when these parameters become insensitive to a further increase in the dip position as shown in Table 1. The empirical relations have been obtained 
TABLE 1: Observations to establish design relationships for ZWF.

\begin{tabular}{|c|c|c|c|c|}
\hline Number of dips, $L$ & Position of dips, $D_{p}$ & Filter order, $N$ & Normalized window width, $D$ & Stopband attenuation, $A_{s}(\mathrm{~dB})$ \\
\hline \multirow{4}{*}{1} & 1 & 17 & 1.576 & 16.93 \\
\hline & 1.1 & 21 & 2.09 & 20.61 \\
\hline & 1.6 & 35 & 3.35 & 38.89 \\
\hline & 1.7 & 37 & 3.61 & 42.57 \\
\hline \multirow{2}{*}{2} & 2 & 47 & 4.53 & 44.12 \\
\hline & 2.6 & 57 & 5.73 & 60.73 \\
\hline \multirow{2}{*}{3} & 3 & 69 & 6.74 & 61.09 \\
\hline & 3.8 & 79 & 7.87 & 76.66 \\
\hline \multirow{2}{*}{4} & 4 & 91 & 8.98 & 78.49 \\
\hline & 4 & 99 & 9.84 & 90.34 \\
\hline \multirow{2}{*}{5} & 5 & 111 & 11.03 & 93.28 \\
\hline & 5 & 121 & 11.95 & 105.67 \\
\hline \multirow{2}{*}{6} & 6 & 131 & 13.04 & 107.82 \\
\hline & 6 & 141 & 13.92 & 118.93 \\
\hline \multirow{2}{*}{7} & 7 & 151 & 15.05 & 121.36 \\
\hline & 7 & 161 & 15.91 & 132.82 \\
\hline \multirow{2}{*}{8} & 8 & 171 & 17.04 & 135.27 \\
\hline & 8 & 181 & 17.91 & 146.78 \\
\hline \multirow{2}{*}{9} & 9 & 191 & 19.05 & 149.31 \\
\hline & 9 & 199 & 19.89 & 160.68 \\
\hline \multirow{2}{*}{10} & 10 & 213 & 21.12 & 163.82 \\
\hline & 10 & 219 & 21.85 & 174.42 \\
\hline
\end{tabular}

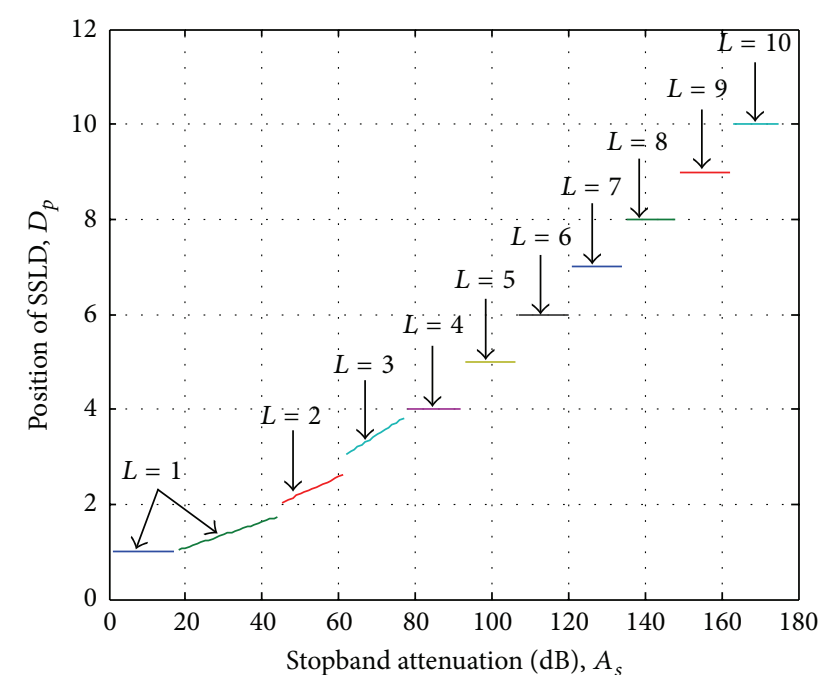

FIgURE 5: Plot of $A_{s}$ versus $D_{p}$ for $Z$ W with different number of dips.

by taking an initial value of $N=17$, normalized passband frequency $\left(f_{p}\right)=0.2$, normalized stopband frequency $\left(f_{s}\right)$ $=0.3$, and sampling frequency $\left(F_{s}\right)=1$. To design the ZWF, dips can be inserted anywhere between $D_{p}$ and $N / 2$. Dips are steered in the vicinity of $D_{p}$ if small TBW is required, while

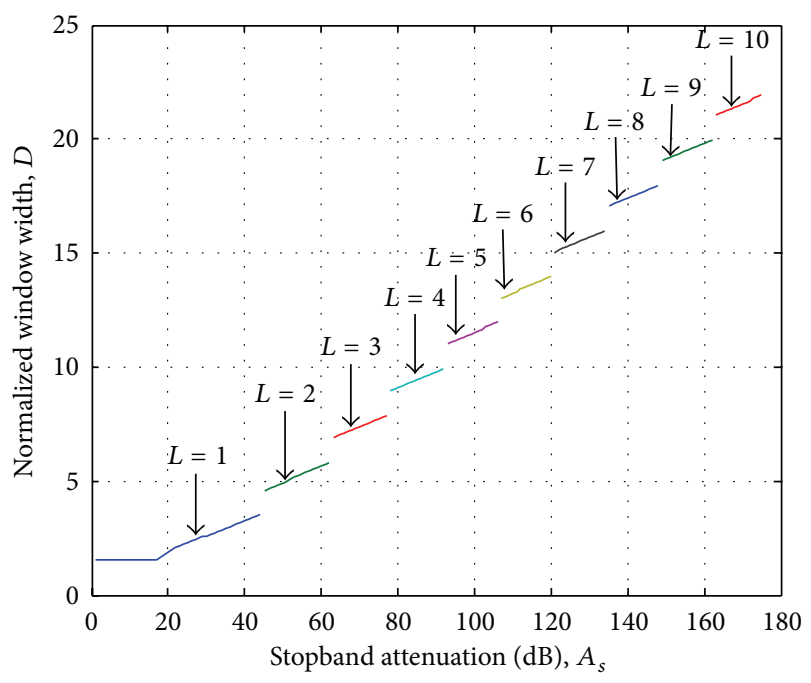

FIgure 6: Plot of $A_{s}$ versus $D$ for ZW with different number of dips.

position of dips is moved towards $N / 2$ if a large FESBA is needed. These design relationships are suitable up to $A_{s}=$ $175 \mathrm{~dB}$ and $L=10$. However, if the desired SBA is more than $175 \mathrm{~dB}$ the number of dips may be increased by one for every $12-15 \mathrm{~dB}$ increment in SBA approximately. The limiting 


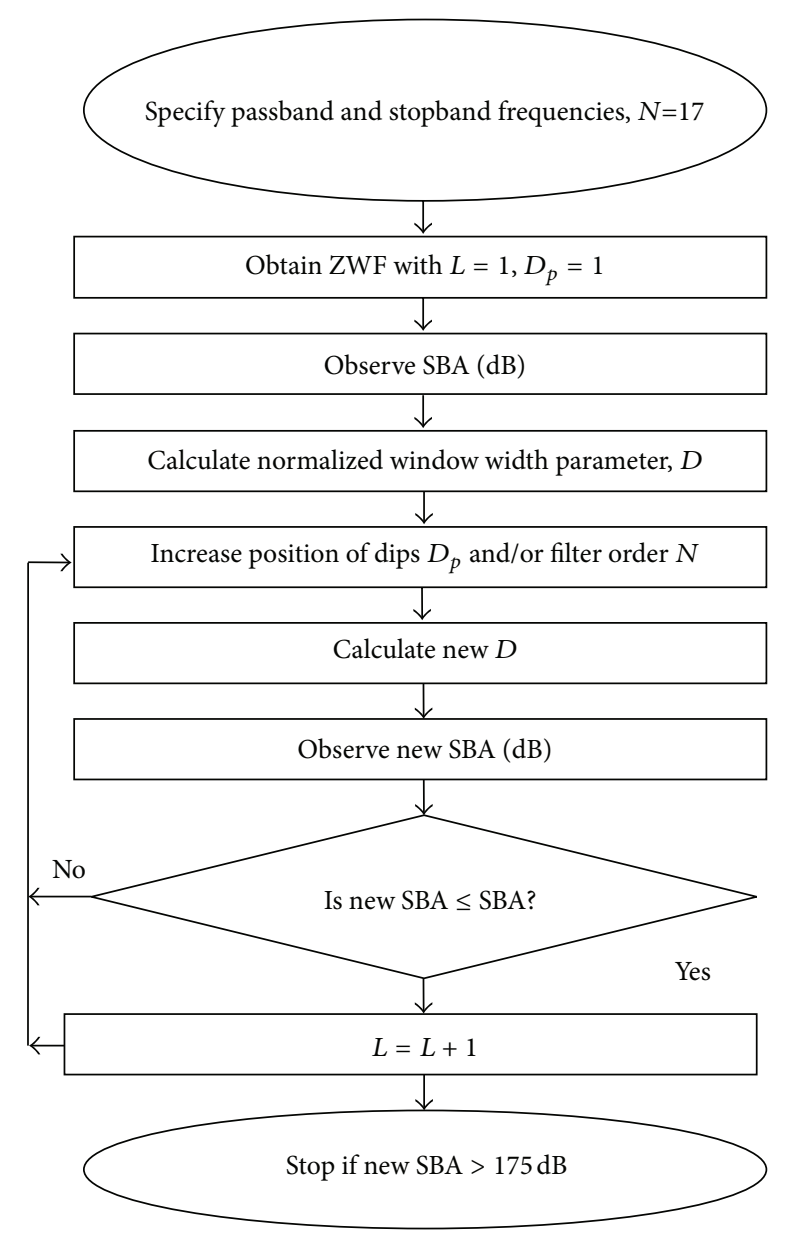

FIGURE 7: Proposed flow chart to obtain the design relationships.

values of SBA range for different number of dips are shown in Table 2. Using Figures 5 and 6 following filter design relationships are obtained:

$$
\begin{gathered}
D_{p}= \begin{cases}1, & A_{s} \leq 17, \\
0.0273 A_{s}+0.5391, & 17<A_{s} \leq 44, \\
0.03612 A_{s}+0.0474, & 44<A_{s} \leq 61, \\
0.05073 A_{s}-0.089, & 61<A_{s} \leq 77, \\
L, & 77<A_{s},\end{cases} \\
D= \begin{cases}1.576, & A_{s} \leq 17, \\
0.0682 A_{s}+0.6824, & 17<A_{s} \leq 44, \\
0.0718 A_{s}-0.6486+L, & 44<A_{s} .\end{cases}
\end{gathered}
$$

TABLE 2: Required number of dips and range of stopband attenuation.

\begin{tabular}{lc}
\hline Number of dips, $L$ & Range of SBA, $A_{s}(\mathrm{~dB})$ \\
\hline 1 & $\leq 44$ \\
2 & $\leq 61$ \\
3 & $\leq 77$ \\
4 & $\leq 92$ \\
5 & $\leq 106$ \\
6 & $\leq 120$ \\
7 & $\leq 134$ \\
8 & $\leq 148$ \\
9 & $\leq 162$ \\
10 & $\leq 175$ \\
\hline
\end{tabular}

These equations have been used to design FIR filters using ZW for a given set of specifications.

\section{Simulation Results and Discussion}

The FIR filters using KW and ZW are simulated for establishing a comparative study as well as for determining the performance of the ZWF. Simulation results are shown in Figures 8,9 , and 10 . ZWFs have been designed with one, two, and three dips. Three sets of different $A_{p}$ and $A_{s}$ (i.e., 1.5 and $23 \mathrm{~dB} / 0.1$ and $50 \mathrm{~dB} / 0.01$ and $74 \mathrm{~dB}$ ) have been used. The order of filter $N$, TBW, and FESBA has been recorded in Table 3 corresponding to these sets of $A_{p}$ and $A_{s}$. ZWF response has been observed at the lower and upper bounds of dip positions, that is, at $D_{p}$ and $N / 2$. To establish a comparative study for every set of $A_{p}$ and $A_{s}$, two KWFs have been designed: (i) with an order to satisfy the design 


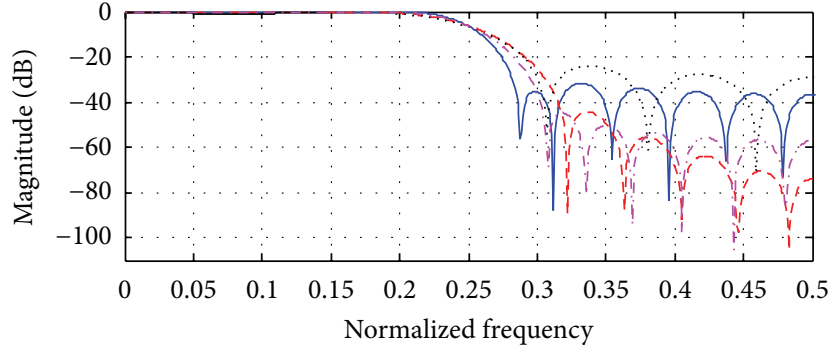

$\begin{array}{llll}\text { Z ZWF, dip at } D_{p} & \cdots & \text { KWF } \\ \ldots- & \text { ZWF, dip at } N / 2 & \ldots & \operatorname{KWF}\left(N_{z}\right)\end{array}$

(a)

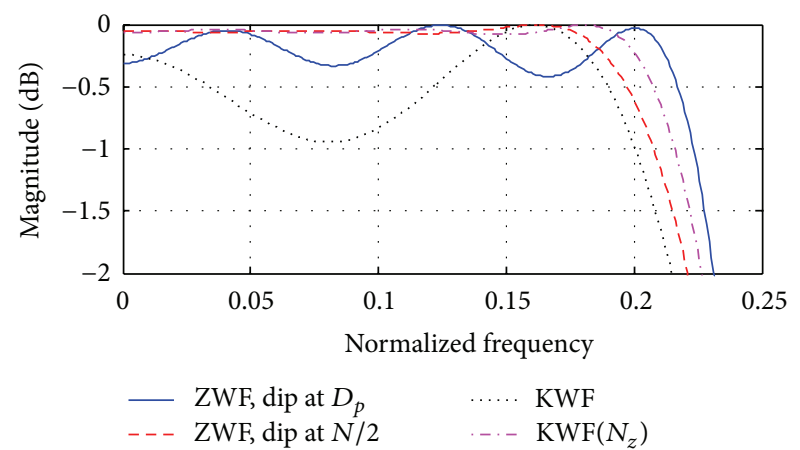

(b)

FIGURE 8: Frequency responses of LPF with $f_{p}=0.2, f_{s}=0.3, A_{p}=1.5 \mathrm{~dB}$, and $A_{s}=23 \mathrm{~dB}$ with insertion of one dip, (a) comparative frequency responses, (b) expanded view of passband.

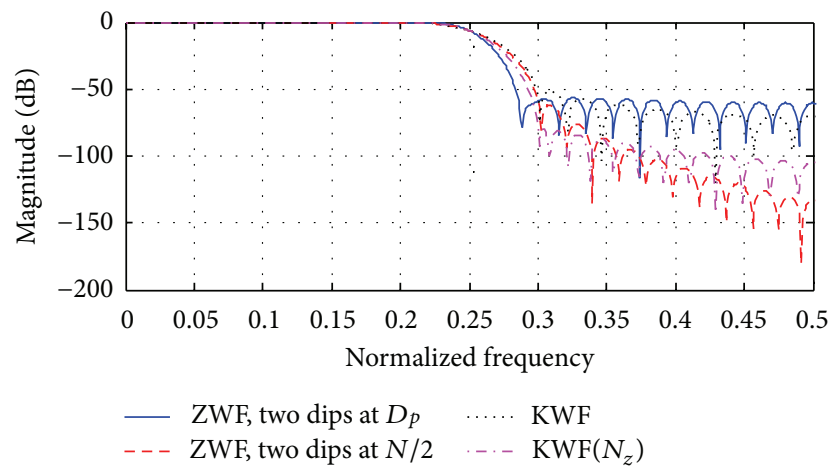

(a)

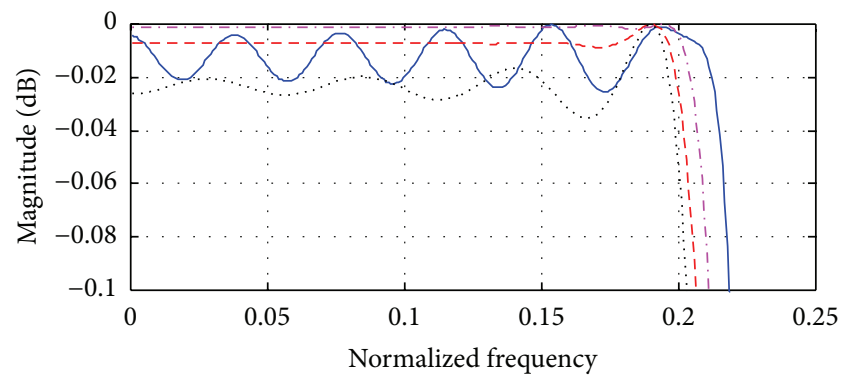

- ZWF, two dips at $D_{p} \quad \cdots \cdots \mathrm{KWF}$ -- ZWF, two dips at $N / 2 \quad \cdots \quad-.-\operatorname{KWF}\left(N_{z}\right)$

(b)

FIGURE 9: Frequency responses of LPF with $f_{p}=0.2, f_{s}=0.3, A_{p}=0.1 \mathrm{~dB}$, and $A_{s}=50 \mathrm{~dB}$ with insertion of two dips, (a) comparative frequency responses, (b) expanded view of passband.

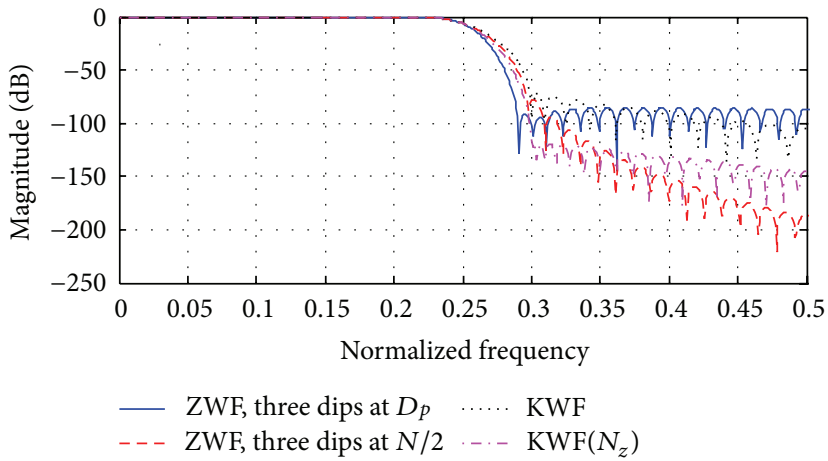

(a)

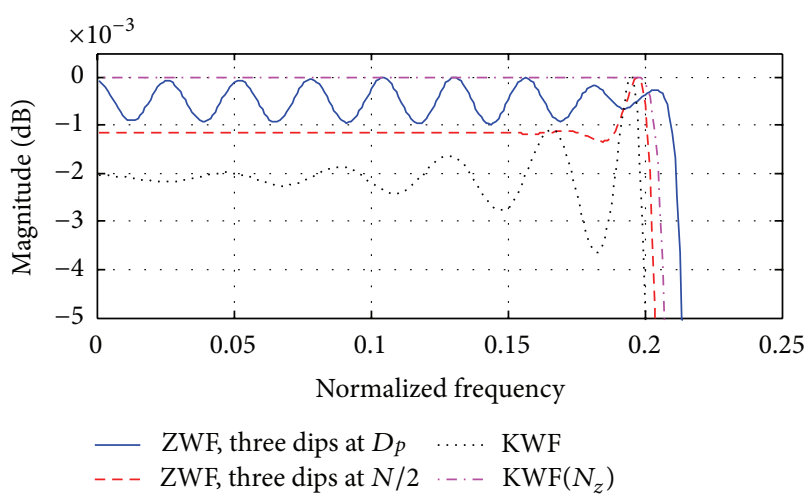

(b)

FIGURE 10: Frequency responses of LPF with $f_{p}=0.2, f_{s}=0.3, A_{p}=0.01 \mathrm{~dB}, A_{s}=74 \mathrm{~dB}$ with insertion of three dips (a) comparative frequency responses (b) expanded view of passband.

specifications and (ii) with an order equal to the ZWF order. From the recorded observations the following conclusions regarding the filters performance have been drawn.

(a) To satisfy desired set of filter specifications a comparatively lower filter order is required by KWF.

(b) With dips steered at $D_{p}$, ZWF has a sharp TBW than KWF for the same filter order. (c) FESBA for a ZWF can be increased by increasing the number of dips and/or by translating the position of dips from $D_{p}$ towards $N / 2$.

(d) For the same filter order ZWF provides greater FESBA than KWF by placing the dips at $N / 2$.

This comparative study established the fact that a tradeoff can be achieved between TBW and FESBA for ZWFs by varying the number of dips and dip positions. To exploit this 
TABLE 3: Comparative performance of LPF.

\begin{tabular}{|c|c|c|c|c|c|c|c|c|}
\hline S. no. & Type of filter & Number of dips, $L$ & Position of dips & Filter order, $N$ & $\begin{array}{l}\text { Desired } \\
A_{p}(\mathrm{~dB})\end{array}$ & $\begin{array}{l}\text { Desired } \\
A_{s}(\mathrm{~dB})\end{array}$ & $\begin{array}{c}\text { Transition } \\
\text { band }\end{array}$ & $\begin{array}{c}\text { FESBA } \\
(\mathrm{dB})\end{array}$ \\
\hline \multirow{4}{*}{1} & ZWF & 1 & $D_{p}$ & 23 & 1.5 & 23 & 0.0497 & -36.59 \\
\hline & ZWF & 1 & $N / 2$ & 23 & 1.5 & 23 & 0.0770 & -74.28 \\
\hline & KWF & - & - & 11 & 1.5 & 23 & 0.0861 & -29.01 \\
\hline & $\operatorname{KWF}\left(N_{z}\right)$ & - & - & 23 & 1.5 & 23 & 0.0652 & -57.20 \\
\hline \multirow{4}{*}{2} & ZWF & 2 & $D_{p}$ & 51 & 0.1 & 50 & 0.0624 & -60.29 \\
\hline & ZWF & 2 & $N / 2$ & 51 & 0.1 & 50 & 0.0891 & -133.1 \\
\hline & KWF & - & - & 31 & 0.1 & 50 & 0.0934 & -78.97 \\
\hline & $\operatorname{KWF}\left(N_{z}\right)$ & - & - & 51 & 0.1 & 50 & 0.0818 & -104.4 \\
\hline \multirow{4}{*}{3} & ZWF & 3 & $D_{p}$ & 77 & 0.01 & 74 & 0.0712 & -86.85 \\
\hline & ZWF & 3 & $N / 2$ & 77 & 0.01 & 74 & 0.0892 & -185.9 \\
\hline & KWF & - & - & 47 & 0.01 & 74 & 0.0988 & -105.4 \\
\hline & $\operatorname{KWF}\left(N_{z}\right)$ & - & - & 77 & 0.01 & 74 & 0.0824 & -145.2 \\
\hline
\end{tabular}

ZWF: ZW based LPF; KWF: KW based LPF.

KWF $\left(N_{z}\right)$ : KW based filter with order of ZWF.

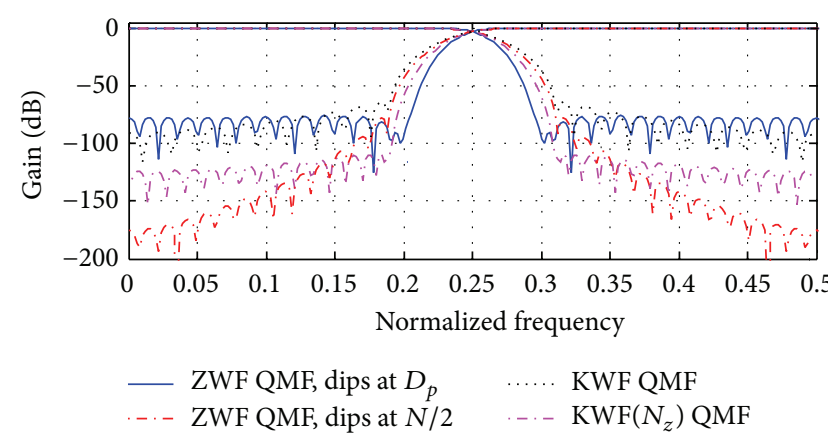

(a)

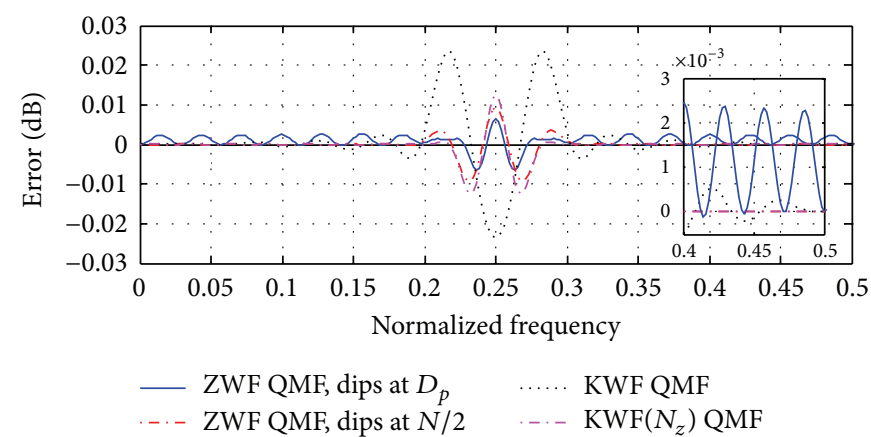

(b)

FIGURE 11: QMF filter banks with $f_{p}=0.15, f_{s}=0.25, A_{p}=0.01 \mathrm{~dB}, A_{s}=60 \mathrm{~dB}$ (a) comparative frequency responses (b) reconstruction error and expanded view of the aliasing error in the inset.

feature of ZWFs they have been used as a prototype filters for QMF banks in the next section.

\section{ZWF Based QMF Bank}

QMF bank has got wide applications in the area of signal processing specially in sub-band coding of speech signal, digital audio applications, communication systems, and short time spectral analysis [15]. In QMF bank, the input signal is split into two equally spaced frequency subbands by twoband analysis filters followed by twofold decimation. At the receiving end, the corresponding synthesis bank has twofold interpolation in both subbands followed by synthesis filters, and finally an adder is used to add both bands. The reconstructed output signal suffers from three types of distortions, that is, aliasing, amplitude or reconstruction, and phase distortion [15]. The aliasing error can be reduced by using a prototype filter with high FESBA, while the reconstruction error can be minimized using filters having sharp transition bands. As in ZW based filters, a tradeoff can be achieved between these two parameters, a QMF bank has been designed with ZWF as a prototype filter, and their performance has been compared with KWF based QMF bank. To demonstrate its usefulness in this multirate signal processing application first lowpass prototype filters have been designed using $\mathrm{ZW}$ and $\mathrm{KW}$ with the following specifications: $f_{p}=0.15, f_{s}=0.25, A_{p}=0.01 \mathrm{~dB}$, and $A_{\text {s }}=60 \mathrm{~dB}$.

Then by using the optimization algorithm proposed by Soni et al. [16, 17] QMF banks have been designed. Corresponding frequency responses and error plots are shown in Figure 11. A comparative study has been carried out in Table 4. From this study it is observed that when dips are translated from $D_{p}$ to $N / 2$ aliasing error decreases. Optimum performance of ZWF based QMF banks has been obtained with dips steered at $N / 2$, providing lowest values of reconstruction and aliasing errors. This performance is better than the KWF based QMF bank designed with the same filter order.

\section{Conclusion}

A study on Z-windows has been carried out in this work by steering number of dips at different positions to characterize the variations in their HMLW, MSLL, and SLFOR. These 
TABLE 4: Comparative performance of QMF bank.

\begin{tabular}{lcccc}
\hline Type of QMF & Filter order, $N$ & Reconstruction error $(\mathrm{dB})$ & Aliasing error $(\mathrm{dB})$ & FESBA $(\mathrm{dB})$ \\
\hline ZWF, dips at $D_{p}$ & 70 & 0.00649 & $2.319 \times 10^{-3}$ & $4.302 \times 10^{-6}$ \\
ZWF, dips at $N / 10$ & 70 & 0.0172 & $2.584 \times 10^{-7}$ & -78.01 \\
ZWF, dips at $N / 6$ & 70 & 0.0127 & $1.614 \times 10^{-7}$ & -123.2 \\
ZWF, dips at $N / 2$ & 70 & 0.00934 & $1.989 \times 10^{-4}$ & -162.5 \\
KWF & 40 & 0.02349 & $1.007 \times 10^{-6}$ & -90.74 \\
KWZ $\left(N_{z}\right)$ & 70 & 0.01243 & & -125.8 \\
\hline
\end{tabular}

window parameters have been chosen, as they proportionately decide the TBW, SBA, and FESBA parameters of filters. Since the number of dips and their positions together tailored the window shape, they have been compositely employed as a window shape parameter for ZW. The relationships (i) window shape parameter versus SBA and (ii) SBA versus $D$ have been established to design a ZW based filter with desired specifications. Performance of ZWFs with different dip positions has been studied and compared with KWFs. For the same filter order ZWF provides a sharp TBW than KWF when dips are inserted at $D_{p}$, but with small FESBA. Translation of dips from $D_{p}$ towards $N / 2$ results in a ZWF with better FESBA than KWF, but at the cost of relatively higher TBW. To exploit this trade-off between TBW and FESBA, ZWF has been used as a prototype filter to design a two-channel QMF bank using the optimization algorithm proposed by Soni et al. $[16,17]$. Comparatively small distortion values in the ZWF based QMF bank have been obtained. Applicability of this filter can be further explored in other multirate signal processing applications.

\section{References}

[1] L. R. Rabiner, J. F. Kaiser, O. Herrmann, and M. T. Dolan, "Some comparisons between FIR and IIR digital filters," Bell System Technical Journal, vol. 53, no. 2, pp. 305-331, 1974.

[2] L. R. Rabiner and B. Gold, Theory and Applications of Digital Signal Processing, Prentice Hall, Englewood Cliffs, NJ, USA, 1975.

[3] F. J. Harris, "On the use of windows for harmonic analysis with the discrete Fourier transform," Proceedings of the IEEE, vol. 66, no. 1, pp. 51-83, 1978.

[4] J. K. Gautam, A. Kumar, and R. Saxena, "Windows: a tool in signal processing," IETE Technical Review, vol. 12, no. 3, pp. 217226, 1995.

[5] F. I. Tseng, T. K. Sarkar, and D. D. Weiner, "A novel window for harmonic analysis," IEEE Transactions on Acoustics, Speech, and Signal Processing, vol. 29, no. 2, pp. 177-188, 1981.

[6] J. Zhong, Z. Han, and W. Lu, "Design of windows with steerable sidelobe dips," IEEE Transactions on Signal Processing, vol. 40, no. 6, pp. 1452-1459, 1992.

[7] S. N. Sharma, R. Saxena, and S. C. Saxena, "Design of narrowband frequency sampling FIR filters using Z-window," Signal Processing, vol. 86, no. 11, pp. 3299-3308, 2006.

[8] R. Pachauri, R. Saxena, and S. N. Sharma, "Design of FIR filters with better performance using Z-window," in Proceedings of the International Conference on Communications, Computing and Control Applications (CCCA '11), Hammamet, Tunisia, March 2011.
[9] L. R. Rabiner, B. Gold, and C. A. McGonegal, "An approach to the approximation problem for nonrecursive digital filters," IEEE Transactions on Audio and Electroacoustics, vol. 18, no. 8, pp. 83-106, 1970.

[10] L. R. Rabiner, "Linear program design of finite impulse response digital filters," IEEE Transactions on Audio and Electroacoustics, vol. 20, no. 4, pp. 280-288, 1972.

[11] D. J. Defatta, J. G. Lucas, and W. S. Hodgkiss, Digital Signal Processing: A System Design Approach, John Wiley \& Sons , 1995.

[12] J. F. Kaiser, "Non-recursive digital filter design using the $\mathrm{I}_{0^{-}}$ Sinh window function," in Proceedings of the IEEE International Symposium on Circuits and Systems Proceedings, pp. 22-23, 1974.

[13] K. M. M. Prabhu and K. Bhoopathy Bagan, "FIR digital filter design with variable parameter window families," Journal of the Institution of Electronic and Radio Engineers, vol. 58, no. 6, pp. 295-298, 1988.

[14] S. N. Sharma, R. Saxena, and S. C. Saxena, "Design of FIR filters using variable window families: a comparative study," Journal of the Indian Institute of Science, vol. 84, no. 5, pp. 155-161, 2004.

[15] P. P. Vaidyanathan, "Multirate digital filters, filter banks, polyphase networks, and applications: a tutorial," Proceedings of the IEEE, vol. 78, no. 1, pp. 56-93, 1990.

[16] R. K. Soni, A. Jain, and R. Saxena, "The design of IFIR prototype filter for cosine modulated filter bank and transmultiplexer," International Journal of Electronics and Communications, vol. 67, no. 2, pp. 130-135, 2013.

[17] R. K. Soni, A. Jain, and R. Saxena, "An optimized design of nonuniform filter bank using variable combinational window function," International Journal of Electronics and Communications, vol. 67, no. 7, pp. 595-601, 2013. 

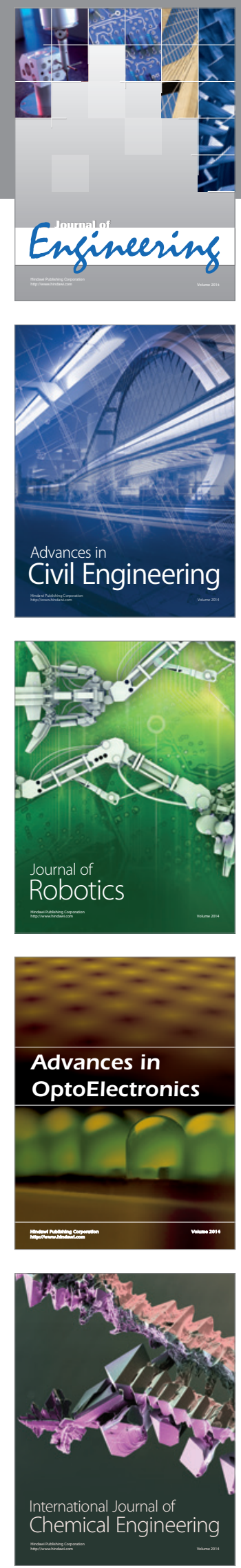

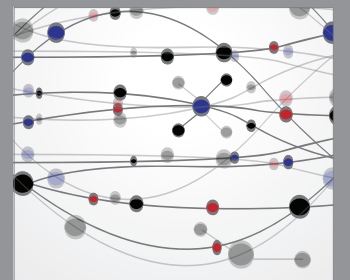

The Scientific World Journal
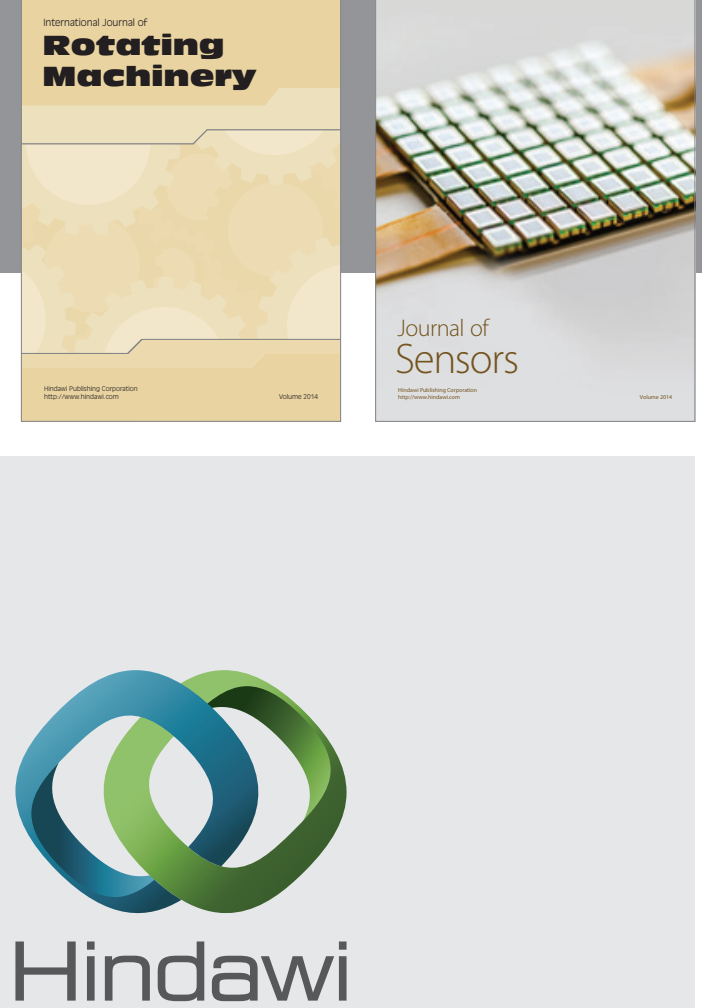

Submit your manuscripts at http://www.hindawi.com
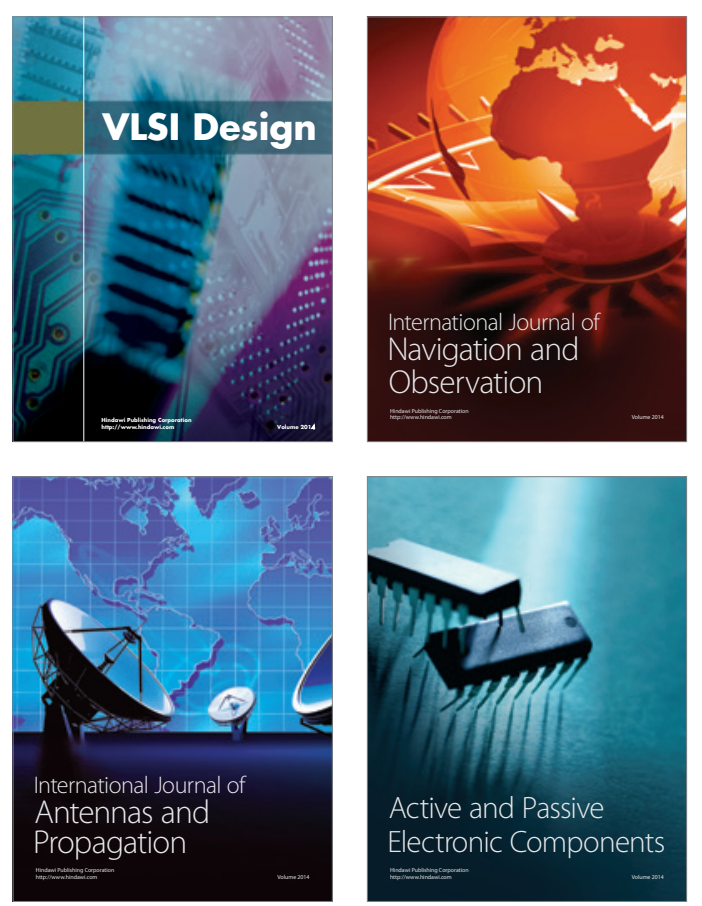
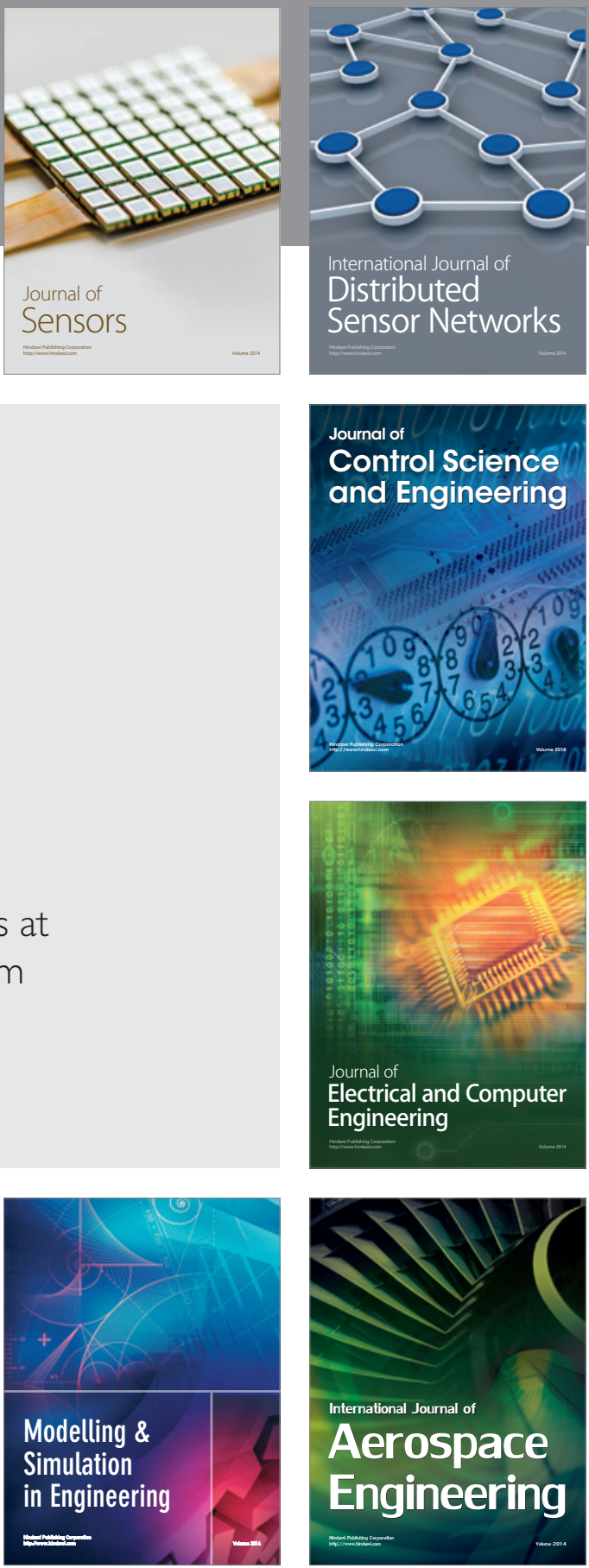

Journal of

Control Science

and Engineering
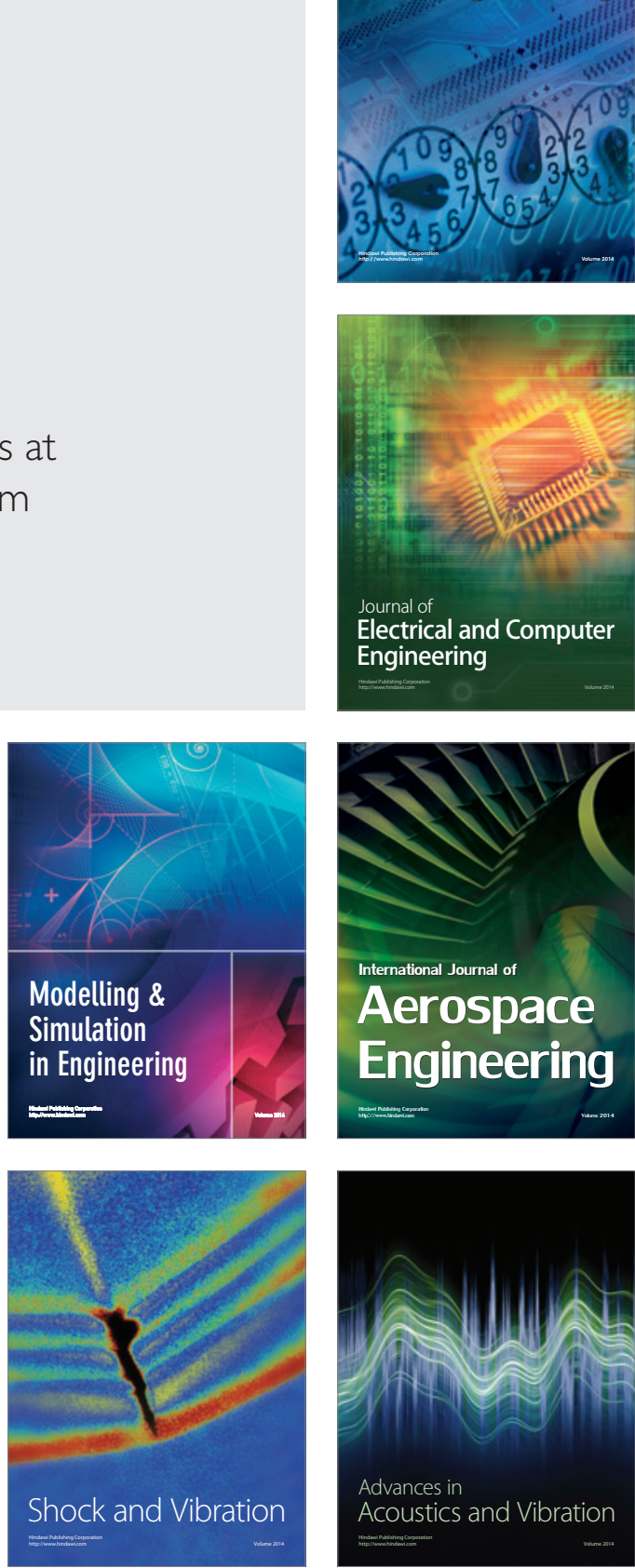